\title{
Influenza and Respiratory Syncytial Virus during the COVID-19 pandemic: time for a new paradigm?
}

Emma Binns ${ }^{1}$, marianne koenraads ${ }^{2}$, Lidia Hristeva ${ }^{3}$, Alix Flamant ${ }^{4}$, Sebastián Baier Grabner $^{5}$, Mervin Loi ${ }^{6}$, Johanna Lempainen ${ }^{7}$, Elise Osterheld ${ }^{8}$, Bazlin Ramly ${ }^{9}$, jessica chakakala-chaziy $^{10}$, Niveditha Enaganthi ${ }^{11}$, Silvia Simó Nebot ${ }^{12}$, and Danilo Buonsenso ${ }^{13}$

${ }^{1}$ Department of Paediatrics, Christchurch Hospital, Christchurch, New Zealand

${ }^{2}$ Paediatric Specialist Trainee, Alder Hey Children's Hospital, Liverpool, UK

${ }^{3}$ General Paediatrics/Neonates, UK

${ }^{4}$ Paediatric Resident, Cliniques Universitaires Saint-Luc, Brussels, Belgium

${ }^{5}$ Department of Paediatrics and Adolescent Medicine, Klinik Ottakring, Vienna Healthcare Group, Vienna, Austria

${ }^{6}$ Children's Intensive Care Unit, KK Women's and Children's Hospital, Singapore

${ }^{7}$ Department of Paediatrics, Institute of Biomedicine and Clinical Microbiology, University of Turku and Turku University Hospital

${ }^{8}$ Department of Paediatrics, Centre Hospitalier de Mayotte, Mayotte, France

${ }^{9}$ Paediatric Department, Children Health Ireland, Dublin, Ireland

${ }^{10}$ Paediatric Department, Muzu Central Hospital, Ministry of Health, Malawi

${ }^{11}$ Paediatric Department,Sri Ramachandra Medical College \& RI ,Chennai,Tamil

Nadu,India

${ }^{12}$ Infectious diseases and systemic inflammatory response in Paediatrics, Infectious Diseases Unit, Sant Joan de Déu Hospital Research Foundation, Barcelona, Spain

${ }^{13}$ Ospedale Pediatrico Bambino Gesu

June 9, 2021

\begin{abstract}
Seasonal epidemics of influenza and the respiratory syncytial virus are the cause of substantial morbidity and mortality among children. During the global COVID-19 pandemic, the epidemiology of these viruses seems to have changed dramatically. In Australia and New Zealand, a significant decrease in both influenza and bronchiolities have been noticed during usual peak seasons. Data from early months of winter seasons in Europe are showing similar trends. This current scenario imposes a reconsideration of the paradigm that toddlers and young schoolchildren are the main drivers of seasonal RSV outbreaks and respiratory epidemics in general. In this paper, we summarize current literature, address current knowledge or role of adults in the respiratory syncitial virus epidemiology, describe the lessons learned from pertussis epidemics and call the international community to better understand the community transmission dynamics of respiratory infections in all age-groups. This can allow the establishment of better and more affordable preventive measures in the whole population level, which can ultimately save millions of child lives.
\end{abstract}

\section{Hosted file}

Manuscript.docx available at https: //authorea.com/users/418812/articles/525455-influenza-and- 
respiratory-syncytial-virus-during-the-covid-19-pandemic-time-for-a-new-paradigm 